\title{
Parasitic Degree Phrases
}

\author{
Jon Nissenbaum \& Bernhard Schwarz \\ McGill University
}

\begin{abstract}
This paper investigates gaps in degree phrases with too, as in John is too rich [for the monastery to hire _ _ ]. We present two curious restrictions on such gapped degree phrases. First, the gaps must ordinarily be anteceded by the subject of the associated gradable adjective. Second, when embedded under intensional verbs, gapped degree phrases are ordinarily restricted to surface scope, unlike their counterparts without gaps. Just as puzzlingly, we show that these restrictions are lifted when there is overt $w h$-movement in the main clause, revealing a striking similarity between the distribution of gapped degree phrases and so-called parasitic gap constructions. These findings, we argue, suggest that the theory of parasitic gaps needs to accommodate gapped degree phrases. Specifically, they argue that parasitic constituents are null operator structures - and under the right conditions need not be accompanied by matrix wh-movement.
\end{abstract}

\section{Keywords}

Degree phrases, scope, gapped infinitival clauses, null operator movement, parasitic gaps

\section{Introduction}

Examples like those in (1) are canonical illustrations of so-called parasitic gaps, featuring an ordinary gap in the main clause, left by a familiar instance of $w h$-movement, and in addition a less ordinary, parasitic, gap in an adjunct or a subject, which is understood as anaphoric to the ordinary gap's antecedent.

(1) a. this article, which $_{\mathrm{i}}$ we filed $\mathrm{t}_{\mathrm{i}}$ [without reading _ ]

b. this theory, which ${ }_{i}$ [people who argue against _ _ often end up adopting $t_{i}$

Despite decades of research on the phenomenon, there remains considerable controversy as to its analysis. The central issue has been the choice between what Nissenbaum (2000) dubbed the "shared antecedent" approach and "separate antecedent" approach. The shared antecedent approach, versions of which have been advocated by Pesetsky (1982), Kayne (1984), Nunes (1995), and Hornstein and Nunes (2002), among others, typically assumes a kind of across-the board wh-movement, as sketched in (2). In contrast, the separate antecedent approach, due to Chomsky (1986), posits separate 
antecedents for the two gaps, making the parasitic gap the trace of a null operator, as sketched in (3). ${ }^{1}$

a. this article, which ${ }_{i}$ [we filed $t_{i}$ ] [without reading $t_{i}$ ]

b. this theory, which $h_{i}$ [people who argue against $t_{i}$ ] [often end up adopting $t_{i}$ ]

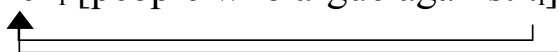

a. this article, which $\mathrm{i}_{\mathrm{i}}$ [we filed $\mathrm{t}_{\mathrm{i}}$ ] $\mathrm{O}_{\mathrm{j}}$ [without reading $\mathrm{t}_{\mathrm{j}}$ ]

b. this theory, which $\mathrm{O}_{\mathrm{j}}$ [people who argue against $\mathrm{t}_{\mathrm{j}}$ ] [often end up adopting $\mathrm{t}_{\mathrm{i}}$ ]

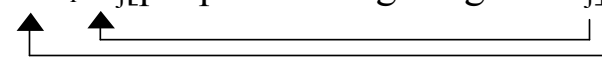

The shared antecedent approach derives in a straightforward way the fact that the parasitic gap relates anaphorically to the ordinary gap's antecedent. As Nissenbaum (2000) shows, the separate antecedent approach can derive this as well, once it is supplemented with suitable assumptions about compositional semantic interpretation. Nissenbaum proposes that in the case of parasitic gaps in adjunct clauses, null operator movement derives a predicate which composes with another predicate through generalized conjunction. This analysis assigns (1a) the semantically interpreted structure in (4). The two gaps in (1a) result from two separate movements, each of which introduces its own lambda binder. Generalized conjunction creates a conjoined predicate, whose argument will be understood as filling each of the two gaps in question, thereby establishing the intended anaphoric connection.

this article, which $[\lambda x[$ we filed $x] \lambda y[$ without reading $y]]$

Nissenbaum shows that this account can be extended to cases like (1b) as well, once a composition principle of generalized functional application is introduced which parallels that of generalized conjunction.

Chomsky (1986) proposed the separate antecedent approach in part to explain asymmetries in the binding of reflexives. As illustrated in (5a), a reflexive in a moved whphrase can be licensed by virtue of the wh-phrase's ordinary trace being c-commanded by a local antecedent. Example (5b), where the reflexive in the moved wh-phrase is not licensed by any potential antecedent in the adjunct, shows that the same does not hold for the parasitic gap. This asymmetry suggests that only the ordinary gap is part of the same chain as the moved wh-phrase.
a. [Which articles about herself[i did Mary read $t_{i}$ [before John filed away]?
b. *[Which articles about herself $]_{i}$ did John read $t_{i}$ [before Mary filed away]?

\footnotetext{
1 The approaches have in common the assumption that parasitic gaps are traces, an assumption which is well-supported by locality considerations (Kayne 1984, Chomsky 1986).
} 
Reflexive binding asymmetries and related asymmetries described in later literature (e.g. Browning 1987, Nissenbaum 2000) may be taken to point to the separate antecedent approach as the correct analysis of parasitic gaps. Unfortunately, however, there are considerations pointing in the opposite direction as well. The separate antecedent approach falls short of deriving what is often considered a defining property of the parasitic gap phenomenon, namely that there must be a movement in the main clause in order for a parasitic gap to appear. This is illustrated by the unacceptability of (6), where in contrast to (1a), no $w h$-movement has occurred in the main clause. ${ }^{2}$

*John filed that article [without our talking to _ ]

Sentence (6) is not expected to be grammatical in the shared antecedent approach. The shared antecedent approach derives in a straightforward way the generalization that movement leaving a trace in the main clause is a precondition on the occurrence of parasitic gaps. ${ }^{3}$ Under the separate antecedent approach, by contrast, it is unclear whether there is a principled answer to the question why a structure like (7) is not well-formed.

John [ [filed that article] $\left[\mathrm{O}_{\mathrm{j}}\right.$ without our talking to $\left.\mathrm{t}_{\mathrm{j}}\right]$ ]

In particular, (7) is not excluded by Nissenbaum's (2000) assumptions about semantic interpretation outlined above. After all, filed that article is a predicate, and so nothing would seem to prevent the gapped adjunct clause from composing with it by way of generalized conjunction. So one would expect (7) to be acceptable and convey that John filed that article without our talking to him.

Aware of this issue, Nissenbaum (2000) suggested that the structure in (7) is excluded as syntactically ill-formed. Specifically, Nissenbaum conjectured that that the illformedness of (7) has to do with the syntactic category of the predicate to which the gapped adjunct attaches. Assuming that filed that article is parsed as V', Nissenbaum suggested that syntax bans adjunction of gapped adjuncts to expressions of that particular category, perhaps to $\mathrm{X}^{\prime}$-level categories in general.

Note that on this view, the unacceptability of (6) is somewhat accidental, in the sense of being due to a syntactic constraint on adjunction, rather than to conditions on movement or the gaps it leaves behind. Accordingly, while excluding (7), the account still falls short of predicting that parasitic gaps must always be licensed by movement in the matrix clause. In order to really replicate this property of the shared antecedent theory, it seems that one would need to directly restrict adjunction of predicates derived by null

${ }^{2}$ Here and throughout we use "main clause" gap to refer to the non-parasitic gap, i.e. the gap that is not embedded in an adjunct clause or in an argument of the main clause predicate.

${ }^{3}$ Strictly speaking, (6) does involve movement in the main clause, if subjects are derived from a VPinternal position. However, John is not expected to be able to move across-the-board in (6), as only one of its traces, the parasitic gap and not the subject trace, would appear in a Case position; independent evidence suggests that across-the-board movement cannot mix A- and A-bar movement in this way. 
operator movement to those that are themselves derived by movement. However, a stipulation of this sort lacks independent empirical support and moreover seems difficult to defend on theoretical grounds. In fact, such a condition would go too far, as it would incorrectly exclude such constructions as relative clauses.

Parasitic gaps, then, present the analyst with the following dilemma. On the one hand, reflexive binding asymmetries and related observations suggest that parasitic gaps are not part of the same chain as a $w h$-phrase that seems to license them. On the other hand, the assumption that parasitic gaps are the tails of their own chains, headed by null operators, falls short of predicting that parasitic gaps need movement in the matrix to be well-formed.

In this paper, we argue that the separate antecedent theory is actually right not to make the latter prediction, and that the shared antecedent is correspondingly wrong in making it. We identify a class of gaps which need not be licensed by matrix $w h$-movement, and we argue that they are nevertheless parasitic. Such non-co-bound "parasitic" gaps constitute exactly the missing case whose apparent absence shed doubt on the separate antecedent approach.

Specifically, we will take cases like (8), which features a non-subject gap in an infinitival associated with the degree operator too, to present cases of acceptable parasitic gaps in the absence of a licensing movement.

Berlin is too cold [for us to travel to __ ]

The gap in (8) has not previously been classified as parasitic, precisely because it is not licensed by a separate movement. Accordingly, one of our main contributions will be a detailed argument that such gaps nevertheless form a natural class with canonical parasitic gaps of the sort illustrated in (1). 4

The argument will be based on certain cases of infinitivals with too which are superficially similar to (8), but differ from it crucially in that a non-subject gap in the infinitival does need to be licensed by movement. We will discuss two kinds of cases of this sort. One kind concerns possible and impossible interpretations when the degree operator too is embedded in an intensional environment. We will show that only when there is an overt main-clause wh-movement can too scope past an intensional verb. The second kind of case concerns possible and impossible antecedents for the gap. We will show that overt main-clause $w h$-movement allows for the gap to enter into otherwise impossible anaphoric relations.

An illustration of the second kind, given in (9) below, will suffice here to support a sketch of our argument. The contrast between the acceptable (8) and the unacceptable (9a) is an instance of a generalization observed in Faraci (1974), according to which a nonsubject gap in an infinitival with too must be anaphoric to the subject of the adjectival

\footnotetext{
${ }^{4}$ In degree phrases, only infinitivals with too and enough allow gaps. Other degree operators, such as comparatives and so...that ..., only combine with finite clauses and never allow gaps. To our knowledge, no explanation for this restriction has been proposed, and in this paper, we likewise leave it unexplained.
} 
phrase that too is in construction with. Example (9b), however, shows that this requirement can be lifted in cases where overt wh-movement occurs in the main clause. There, the gap under consideration is anaphoric to which city.
a. *It's too cold in Berlin [for us to travel to
b. [Which city $]_{i}$ is it too cold in $t_{i}$ [for us to travel to ]?

Putting aside examples like (8), in which the gap's antecedent is the subject of the adjective (and the degree operator takes surface scope), gapped degree phrases show the very same distributional characteristics as canonical parasitic gaps. It seems inescapable, then, to analyze gaps like the second one in (9b) as being of the same kind as canonical parasitic gaps. The contrast in (9) is then parallel to the contrast in (10), which involves canonical parasitic gaps.
a. *He traveled to Berlin [to take pictures of _ ]
b. [Which city $]_{i}$ did he travel to $t_{i}[$ to take pictures of __ ]?

This in principle still leaves open the possibility that the gap in (8) is not parasitic, leading to a disjunctive analysis of gaps in infinitivals with too-i.e. where cases like (9b) analyzed as parasitic while those like (8) are not. However, we will argue that such an approach would leave us without an explanation for the restrictions described above, namely the requirement that (absent matrix wh-movement) gaps in infinitivals with too must be anaphoric to the adjective's subject, and must take surface scope.

If we are correct, then the proper theory of parasitic gaps must have examples like (8) in its scope and therefore should not categorically exclude parasitic gaps in the absence of a licensing movement. By the same token, it follows that cases like (6) should not be excluded by the theory of parasitic gaps itself, but by independent syntactic constraints, such as a ban against attachment to $\mathrm{V}^{\prime}$.

If accepted, then, our argument resolves the dilemma described above, strengthening the case for a separate antecedent account of parasitic gaps, and excluding the shared antecedent approach.

In section 2, we will begin spelling out the argument outlined above by providing a preliminary syntactic and semantic analysis of the degree operator too, building on Chomsky (1977) and Heim (2001). In section 3, we present constraints on gapped infinitivals with too. We show that under the preliminary analysis introduced in section 2 , these constraints would follow from the assumption that too plus gapped infinitival must be interpreted in situ. We also note, however, that this assumption does not seem motivated on independent grounds. In section 4, we develop an alternative picture under which gapped degree phrases are analyzed just like adjunct clauses and DPs hosting parasitic gaps are analyzed under the separate antecedent approach, positing null operator movement to the edge of the degree phrase. We suggest that the restrictions identified in section 3 are instances of syntactic constraints limiting the distribution of parasitic gaps. In section 5 we show that a surprising prediction of our alternative theory is borne out, 
namely that the constraints identified in section 3 are lifted in precisely the environments where canonical parasitic gaps are licensed. In section 6 we explain in detail how our findings support the separate antecedent theory of parasitic gaps.

\section{A first analysis of too plus gapped infinitival}

We have seen that the degree operator too can combine with an infinitival clause containing a gap in non-subject position. ${ }^{5}$ However, it is not necessary for an infinitival with too to contain a gap. Sentence (11b), for example, is no less acceptable than sentence (11a). In fact, reading the object pronoun him in (11a) as anaphoric to John, (11a) and (11b) are synonymous.

(11) a. John is too rich [for the monastery to hire him].

b. John is too rich [for the monastery to hire _ ] .

How do sentences like those in (11) get to be synonymous? Naturally, the answer to this question depends in part on what the source of gap in (11b) is taken to be. Chomsky (1977) argued for an approach in which the non-subject gap in an infinitival with too is a trace left behind by a phonetically null operator that has moved to the edge of its clause to form a predicate of individuals. Chomsky also suggested that the infinitival is a complement of too. Under this assumption, a straightforward extension of Chomsky's proposal could account for the alternation in (11) by positing two homophonous degree operators too, one that takes a propositional infinitival clause as its complement and one that combines with a property-denoting infinitival. In the remainder of this section, we will spell out such an analysis. ${ }^{6}$

\subsection{Letting too Fill the Gap}

Based on standard assumptions about gradable adjectives, we formulate in this subsection a semantics for too with non-gapped infinitivals that essentially follows Heim (2001).

Extending this account, we then spell out analysis of too with gapped infinitivals based on Chomsky's (1977) suggestion that a non-subject gap in an infinitival with too is due to null

\footnotetext{
5 In this paper do not address null subjects in infinitival clauses, which are freely available. Henceforth we will often refer to the more restricted non-subject gaps, with which we are concerned, as simply 'gaps'.

6 We note that examples with the degree operator enough raise much the same questions as examples with too. As illustrated in (i), enough also combines with an infinitival clause that may or may not contain a nonsubject gap.
}

(i) a. John is rich enough [for the monastery to hire him].

b. John is rich enough [for the monastery to hire _ ].

Putting aside the obvious difference in meaning between too and enough, everything we have to say about too plus infinitival applies to enough plus infinitival as well. For ease of presentation, however, we will confine our discussion to examples with too. 
operator movement to the edge of the infinitival.

We take gradable adjectives to relate degrees and individuals, following Cresswell (1976), von Stechow (1984), and Heim (2001), among others. Specifically, we assign gradable adjectives denotations of type $\mathrm{d}(\mathrm{e}(\mathrm{st}))$. This is illustrated in the lexical entry in (12), where $\mathrm{RICH}_{\mathrm{W}}(\mathrm{x})$ refers to x's net worth in the possible world w.

$$
\llbracket \text { rich } \rrbracket=\lambda d_{d} \cdot \lambda x_{e} \cdot \lambda w_{s} \cdot \operatorname{RICH}_{w}(x) \geq d
$$

The lexical entry in (12) follows authors like Gawron (1995) and Heim (2001) in letting rich relate a degree $\mathrm{d}$ to an individual just in case the individual's net worth is at least $\mathrm{d}$, as opposed to exactly $\mathrm{d}$. So we take rich to relate a given individual $\mathrm{x}$ not only to x's net worth itself, but also to every degree on the scale of wealth below x's net worth.

The degree operator too has a modal component. Sentence (11a), repeated in (13), has the truth conditions in (14). Taking w to refer to the actual world, (14) conveys that in no accessible world where John is as rich as he actually is does the monastery hire him.

(13) John is too rich [for the monastery to hire him].

(14) $\exists d\left[\operatorname{RICH}_{\mathrm{w}}(\mathrm{j}) \geq \mathrm{d} \& \sim \exists \mathrm{w}^{\prime}\left[\mathrm{w}^{\prime} \in \mathrm{Acc}_{\mathrm{w}} \& \mathrm{RICH}_{\mathrm{w}}(\mathrm{j}) \geq \mathrm{d} \& \mathrm{~m}\right.\right.$ hires j in $\left.\left.\mathrm{w}^{\prime}\right]\right]$

What worlds count as accessible in general depends on context. In the example at hand, the accessible worlds could be those worlds where the monastery's actual hiring policy is obeyed. (14) then says that in no such world where John is as rich as he actually is does the monastery hire him. This is true, for example, if John is a millionaire and the monastery's policy is not to hire anyone whose net worth exceeds a half a million dollars. Note also that (13) is understood to entail that the monastery will not hire John. This can be credited to the assumption that accessibility relation in question is realistic, which entails that the monastery respects its hiring policy in the actual world. ${ }^{7}$

Following Heim (2001), we assign (13) a L(ogical) F(orm) like (15), where too and its infinitival form a degree phrase that has undergone covert movement to the edge of the clause. The trace of this degree phrase is interpreted as a variable ranging over degrees. The truth conditions in (14) can be derived by assigning too the lexical entry in (16), which is close to the one given in Heim (2001).

(15) $\quad[$ too [for the monastery to hire him] $] \lambda \mathrm{d}[\mathrm{John}$ is $\mathrm{d}$ rich]

(16) $\llbracket$ too $\rrbracket=\lambda p_{s t} \cdot \lambda f_{d(s t)} \cdot \lambda w_{s} . \exists d\left[f(d)(w) \& \sim \exists w^{\prime}\left[w^{\prime} \in A c c_{w} \& f(d)\left(w^{\prime}\right) \& p\left(w^{\prime}\right)\right]\right]$

We are now ready to turn to (11b), repeated in (17), which is judged to share with (13) the truth conditions in (14). As mentioned, Chomsky (1977) analyzes the gap in a case like (17) as the trace of a null operator that has moved to the edge of its clause to form a

\footnotetext{
7 Further details of the semantics of too (and enough), orthogonal to the concerns of this paper, are discussed in Hacquard (2006), Meier (2002), Schwarzschild (2008), and von Stechow, Krasikova, and Penka (2004).
} 
predicate of individuals. Following this approach, we can posit (18) as the logical form of (17) as well as the lexical entry in (19).

(17) John is too rich [for the monastery to hire _ ]

(18) John is [too' $\lambda x[$ for the monastery to hire $\mathrm{x}]]$ rich

(19) 【 too' $\rrbracket=\lambda \mathrm{P}_{\mathrm{e}(\mathrm{st})} \cdot \lambda \mathrm{F}_{\mathrm{d}(\mathrm{e}(\mathrm{st}))} \cdot \lambda \mathrm{x}_{\mathrm{e}} \cdot \lambda \mathrm{w}_{\mathrm{s}} \cdot \exists \mathrm{d}\left[\mathrm{F}(\mathrm{d})(\mathrm{x})(\mathrm{w}) \& \sim \exists \mathrm{w}^{\prime}\left[\mathrm{w}^{\prime} \in \mathrm{Acc}_{\mathrm{w}} \& \mathrm{~F}(\mathrm{~d})(\mathrm{x})\left(\mathrm{w}^{\prime}\right)\right.\right.$ $\left.\& \mathrm{P}(\mathrm{x})\left(\mathrm{w}^{\prime}\right)\right]$ ]

In (18), the degree phrase remains in situ, hence below the subject, and it is headed by a too', a type shifted homophone of too. The lexical entry in (19) lets too' feed the subject denotation in (18) as an input to the property denoted by the gapped infinitival (as well as to the degree relation in the scope of the degree phrase). This ensures that the gap in the infinitival is anaphoric to the subject. It thereby ensures that (18) is indeed assigned the intended truth conditions in (14).

We consider this analysis of too plus gapped infinitivals an obvious extension of the proposal in Chomsky (1977). What we will now show, however, is that too plus gapped infinitival is subject to restrictions that the analysis does not lead one to expect.

\section{Unexpected restrictions}

In this section we present two kinds of restrictions on too plus gapped infinitival. First, in combination with a gapped infinitival the degree operator too lacks certain scope options otherwise available to it. Second, a non-subject gap in an infinitival with too is required to be anaphoric to the subject argument of the associated gradable adjective. ${ }^{8}$ We describe each of these restrictions below and we show that under the account in section 2, they indicate that the degree phrase formed by too and its infinitival must be interpreted in situ. We also argue that under the analysis under consideration, such an in situ restriction cannot be motivated on independent grounds.

\subsection{Frozen Scope}

Heim (2001) observes that under (certain) intensional verbs, degree phrases with too participate in an ambiguity that can be analyzed as an ambiguity of logical scope. To illustrate, consider sentence (20), where the degree phrase headed by too is embedded under want.

John wants to be too rich [for the monastery to hire him].

Consider the LF for (20) shown in (21), where the degree phrase takes scope within the embedded clause. Assuming that PRO refers to John, the truth conditions assigned to this LF are those shown in (22). (22) conveys that in all of John's desire worlds he is too rich

\footnotetext{
${ }^{8}$ Actually, each of the two restrictions has exceptions, but we will set them aside until section 5 .
} 
for the monastery to hire. In this predicted reading, then, the sentence entails that John wants not to be hired.

(21) John wants [ [too [for the monastery to hire him] ] $\lambda \mathrm{d}[\mathrm{PRO}$ be d rich] ]

(22) $\forall \mathrm{w}^{\prime \prime}\left[\mathrm{w}^{\prime \prime} \in \mathrm{Bul}_{\mathrm{w}}(\mathrm{j}) \rightarrow \exists \mathrm{d}\left[\operatorname{RICH}_{\mathrm{w}}(\mathrm{j}) \geq \mathrm{d} \& \sim \exists \mathrm{w}^{\prime}\left[\mathrm{w}^{\prime} \in \operatorname{Acc}_{\mathrm{w}^{\prime \prime}} \& \mathrm{RICH}_{\mathrm{w}}(\mathrm{j}) \geq \mathrm{d} \& \mathrm{~m}\right.\right.\right.$ hires j in $\left.\mathrm{w}^{\prime}\right]$ ] ]

To be sure, sentence (20) can indeed be understood in this way. However, the sentence also has another reading, one that is consistent with John having no objection to being hired by the monastery and that could even be true if John wants them to hire him. The relevant reading of the sentence would be salient in a scenario where the monastery's hiring policy makes reference to applicants' desired wealth, rather than their actual wealth, excluding every candidate whose desired net worth is a above a certain limit. Sentence (20) could then be understood as conveying that John's desired net worth is above that limit. In other words, (20) seems to have a reading with the truth conditions in (23).

$$
\begin{aligned}
& \exists d\left[\forall w ^ { \prime \prime } [ w ^ { \prime \prime } \in B u l _ { w } ( j ) \rightarrow \operatorname { R I C H } _ { w ^ { \prime \prime } } ( j ) \geq d ] \& \sim \exists w ^ { \prime } \left[w ^ { \prime } \in A c c _ { w } \& \forall w ^ { \prime \prime } \left[w^{\prime \prime} \in B u w_{w^{\prime}}(j) \rightarrow\right.\right.\right. \\
& \left.\mathrm{RICH}_{w^{\prime \prime}}(\mathrm{j}) \geq \mathrm{d} \text { ] \& } \mathrm{m} \text { hires j in } w^{\prime}\right] \text { ] ] }
\end{aligned}
$$

The availability of such a reading is precisely what one expects if, as Heim (2001) argues, degree phrases with too can take inverse scope over (certain) intensional verbs. That is, the truth conditions in (23) can be credited to the LF (24), where the degree phrase covertly moves from the embedded clause to take widest scope.

$$
\text { [too [for the monastery to hire him] }] \lambda d[\text { John wants [PRO be } \mathrm{d} \text { rich] }]
$$

Consider now sentence (25) below, which minimally differs from (20) in that the object pronoun in the infinitival clause accompanying too is omitted. The relevant observation, previously unnoticed, is that (25) does not share with (20) the ambiguity described above. While the sentence allows for the reading in (22), it is judged to lack the one in (23). So in contrast to (20), sentence (25) unambiguously entails that John does not want to be hired.

John wants to be too rich [for the monastery to hire _ _ ].

Under the analysis given above, the availability of reading (22) indicates that it is possible for the degree phrase headed by too' to be interpreted in situ, in the scope of want, but that this degree phrase cannot take inverse scope over want. So intuitions on (25) indicate that while the LF (26) is available, the one in (27) is not. ${ }^{9}$

9 In the unavailable LF (27), the degree phrase would adjoin just below the subject, in either its underlying or its derived position. In this section we will not distinguish between the two possible landing sites, but they will become relevant in section 4 . 
John wants [PRO be [too' $\lambda x$ [for the monastery to hire $\mathrm{x}]$ ] rich] John [too' $\lambda x[$ for the monastery to hire $\mathrm{x}]$ ] $\lambda \mathrm{d}$ [wants [PRO be $\mathrm{d}$ rich] ]

It is the unavailability of (27) that comes as a surprise. What is it that keeps a degree phrases with too from taking inverse scope if it combines with a gapped infinitival clause?

We begin to address this question in subsection 3.3, where we introduce another type of restriction on too plus gapped infinitival, first described in Faraci (1974). The restriction in question does not concern the relative scope of the degree phrase and another operator, but the possible antecedents of non-subject gaps in infinitivals with too.

However, in the next subsection we first return to sentence (20). As the reader may have noticed, the two reading described above do not exhaust the range of interpretations available to the sentence.

\subsection{Aside: A Third Reading}

We have so far identified two interpretations of sentence (20). The first reading is characterized by the entailment that John wants not to be hired by the monastery. The second reading lacks this entailment and suggests an inconsistency between the monastery's hiring policy and John's desired wealth.

But there appears to be a third way of understanding the sentence. Consider the following scenario: John wants to be a millionaire; he also wants to be hired by the monastery; however, the monastery's hiring policy imposes conditions on applicants' actual degree of wealth - as opposed to their desired degree of wealth — and it specifically precludes millionaires from being hired. Neither of the two readings discussed above is true in this scenario. The first reading is false because John wants the monastery to hire him, and the second is false because the monastery's policy imposes no restrictions on desired wealth. Yet it seems that (20) can be judged true in this scenario, indicating that the sentence has a third reading.

A tentative characterization of this reading is given in (28), which differs from (22) above only in the world argument of the accessibility relation $A c c$. In (22) this argument is bound, whereas it picks out the actual world $\mathrm{w}$ in (28).

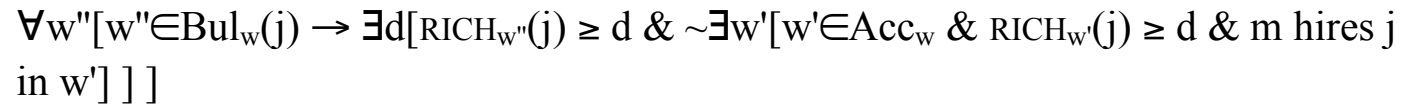

Accordingly, (28) states that in all of John's desire worlds, he is rich to a degree such that his being rich to that degree happens to be inconsistent with the monastery's actual hiring policy, a statement which is true in the scenario described above.

Curiously, sentence (25), the gapped counterpart of (20) does not seem to allow for the reading in (28) any more than it does for the inverse scope reading in (23). As we have reported above, (25) seems to unambiguously entail that John does not want to be hired. 
This raises the obvious questions where reading (28) of sentence (20) is coming from and why this reading would be unavailable for (25).

At this point, we cannot give definite answers to these questions. We believe, however, that the correct answers will not choose between the two analyses of gapped degree phrases we consider in this paper. We refer the interested reader to Nissenbaum and Schwarz (2008) for a proposal about the source of the third reading. In this paper, we will not be concerned any further with the reading in question, focusing instead on the surface and inverse scope readings described in the previous subsection.

\subsection{Faraci's Generalization}

Faraci $(1974,188-9)$ observed that the distribution of gapped infinitivals with too is much more restricted than that of gapless infinitivals. For example, Faraci judges the examples in (29) to be unacceptable.
a. *Mary runs too fast [for me to keep up with _ ].
b. *Homer eats too much [for Jim to keep up with _ _ ].

Faraci demonstrates that the unacceptability of these cases is indeed tied to the presence of the gap. He reports that the sentences in (30), where the gaps are filled with overt pronouns anaphoric to the matrix subject, are fully grammatical.

(30) a. Mary runs too fast [for me to keep up with her].

b. Homer eats too much [for Jim to keep up with him].

Faraci takes these observations to show that a non-subject gap in an infinitival with too can only be anaphoric to the subject argument of the adjective that too combines with. This captures the fact that (29a-b) cannot mean what (30a-b) mean, since in (30a-b) the final pronoun is understood to be anaphoric to the subject of the main predicate (run or eats) rather than the adjective combining with too (fast or much). Moreover, since the adjectives in question do not predicate subjects, at least none that could conceivably antecede the gap in the infinitival, Faraci's generalization correctly excludes the examples in (29) as unacceptable.

Faraci's generalization also applies correctly to cases where the adjective combining with too is the main predicate, but features an internal argument or adjunct. In the grammatical sentence (31), for example, the gap can be understood anaphoric to John, the subject argument of angry, but not to its complement Mary. And sentence (32), which repeats (9a) from the introduction, is expectedly ungrammatical, given that the adjective's expletive subject cannot be interpreted as the antecedent of the gap in the infinitival.

(31) John is too angry at Mary [for us to invite ].

*It's too cold in Berlin [for us to travel to 
Under the assumption we have introduced above, Faraci's generalization comes as a surprise. Assuming that degree phrases with $t o o^{\prime}$ can move covertly just as the gapless variants with too can, one expects (29a-b) to have the LFs in (33a-b), where in each case the degree phrase has landed just below the matrix subject and which would derive the very readings expressed by the non-gapped examples in (30a-b).

a. Mary [too' $\lambda \mathrm{x}[$ for me to keep up with $\mathrm{x}]] \lambda \mathrm{d}[$ runs $\mathrm{d}$ fast]

b. Homer [too' $\lambda x[$ for Jim to keep up with $\mathrm{x}]] \lambda \mathrm{d}[$ eats $\mathrm{d}$ much]

Similarly, unattested readings of (31) and (32) could be derived from the LFs in (34), where Mary or Berlin has covertly moved to the edge of the clause and the too' degree phrase has moved to a position just above the lambda-binder introduced by this covert movement, ensuring that Mary and Berlin, respectively, fills the gap in the infinitival. ${ }^{10}$

$$
\begin{aligned}
& \text { Mary [too' } \lambda \mathrm{x}[\text { for us to invite } \mathrm{x}]] \lambda \mathrm{d} \lambda \mathrm{y}[\mathrm{John} \text { is } \mathrm{d} \text { [angry at } \mathrm{y}]] \\
& \text { Berlin [too' } \lambda \mathrm{x}[\text { for us to travel to } \mathrm{x}]] \lambda \mathrm{d} \lambda \mathrm{y}[\text { it is [ }[\mathrm{d} \text { cold }] \text { [in } \mathrm{y}]
\end{aligned}
$$

Each of the unavailable LFs in (33) to (35) assumes that degree phrases with too' can move covertly. To account for Faraci's generalization, we would accordingly have to assume that degree phrases with too' are not in fact scopally mobile and instead must always be interpreted in situ.

It is apparent that this assumption would also derive the frozen scope observation presented in the previous section. If degree phrases with too' cannot move covertly, then in particular they cannot take inverse scope over higher operators at LF.

The question is why it would be that too plus gapped infinitival has to be interpreted in situ. In section 4, we will develop an alternative analysis which analyzes gapped degree phrases as parasitic gaps. First, however, we will establish a negative result. In the next subsection we show that the constraints identified above are specific to gapped degree phrases with too, as opposed to being general constraints on a wider class of degree operators.

\subsection{A more general constraint?}

One might hope that an in situ restriction on too with a gapped infinitival can be seen as a

10 The LFs in (34) and (35) crucially rely on the view, introduced in Heim and Kratzer (1998) and adopted throughout this paper, that movement introduces a lambda-binder which is prefixed to the moved expression's scope, binding the trace left behind by the moved expression. In a more conventional syntax of chain formation, the function of the lambda-binder is taken by an index that forms a constituent with the moved expression itself, leaving the trace free within the moved expression's scope and leaving the semantic type of the scope unaltered. The conventional syntax would not allow for interpretable LFs corresponding to (34) and (35), where lambda abstraction introduced by one movement creates a suitable landing site for another movement. That lambda abstraction in the syntax is needed has recently been argued by a number of authors (Barker 2007, Beck 2000, Bhatt and Takahashi 2008, Heim 1999, Nissenbaum 1998, Sauerland 1998), based on cases similar in structure to those discussed in this paper. 
more general restriction on a certain type of degree phrases. Specifically, one might hope that this constraint applies to all two-place degree phrases, that is, degree phrases which, like degree phrases with too' and unlike degree phrases with too, combine an individual argument with a denotation of the gradable adjective type. We examine two types of degree phrases that have been given such two-place analyses, namely superlatives (Heim 1999, 2001) and reciprocal equatives (Schwarz 2007). We will see that neither of these cases shows the peculiar scope restriction seen in degree phrases with gapped infinitivals.

In the analysis given in Heim $(1999,2001)$, sentence (36a) has a LF like (36b), where the superlative operator -est occurs in situ. The lexical entry in (37) makes degree phrases with -est two-place in the sense introduced above. This lexical entry renders (36b) interpretable and assigns it the truth conditions in (38), which states that John reaches a degree of wealth that no one else reaches.

$$
\begin{aligned}
& \text { a. John is richest. } \\
& \text { b. John [-est rich] } \\
& \llbracket \text {-est } \rrbracket=\lambda F_{d(e(s t))} \cdot \lambda x_{e} \cdot \lambda w_{s} \cdot \exists d[F(d)(x)(w) \& \sim \exists y[y \neq x \& F(d)(y)(w)] \\
& \exists d\left[R_{C I C H}(j) \geq d \& \sim \exists y\left[y \neq j \& \operatorname{RICH}_{w}(j) \geq d\right]\right.
\end{aligned}
$$

Moving away from simple predicative cases like (36a), consider now the examples in (39). In the relevant readings, these sentences convey that Mary runs fast to a degree that no other relevant person does, and that Berlin is cold to a degree that no other relevant place is, respectively.
a. Mary runs fastest.
b. It is coldest in Berlin.

Under the analysis under consideration, these readings are to be credited to the LFs in (40). These LFs are transparently parallel to the unavailable LFs in (33a) and (35). In (40a), degree phrase movement targets a position right below the subject and in (40b), Berlin has moved covertly, and the degree phrase lands between Berlin and the abstraction its movement has introduced.
a. Mary est $\lambda \mathrm{d}[$ runs $\mathrm{d}$ fast]
b. Berlin est $\lambda \mathrm{d} \lambda \mathrm{x}[$ it is $\mathrm{d}$ cold in $\mathrm{x}]$

Under the analysis given, then, a superlative degree phrase need not be interpreted in situ and in fact allows for the very movement that Faraci's Generalization shows to be unavailable for degree phrases with too'.

The same is true for reciprocal equatives under the analysis given in Schwarz (2007). This analysis assigns example (41a) a LF like (41b). Under the two-place lexical entry for equally in (42), (41b) receives the truth conditions in (43), which amounts to 
saying that John is exactly as rich as Mary.

(41) a. John and Mary are equally rich.

b. [John and Mary] [equally rich]

$$
\begin{aligned}
& \text { 【e equally } \rrbracket=\lambda F_{d(e(s t))} \cdot \lambda z_{\mathrm{e}} \cdot \lambda \mathrm{w}_{\mathrm{s}} \forall \mathrm{x}, \mathrm{y}[\mathrm{x}, \mathrm{y} \leq \mathrm{z} \& \mathrm{x}, \mathrm{y} \in \text { Atom } \rightarrow\{\mathrm{d}: \mathrm{F}(\mathrm{d})(\mathrm{x})(\mathrm{w})\}= \\
& \{\mathrm{d}: \mathrm{F}(\mathrm{d})(\mathrm{y})(\mathrm{w})\}] \\
& \forall \mathrm{x}, \mathrm{y}\left[\mathrm{x}, \mathrm{y} \leq \mathrm{j}+\mathrm{m} \& \mathrm{x}, \mathrm{y} \in A \text { tom } \rightarrow\left\{\mathrm{d}: \mathrm{RICH}_{\mathrm{w}}(\mathrm{j}) \geq \mathrm{d}\right\}=\left\{\mathrm{d}: \mathrm{RICH}_{\mathrm{w}}(\mathrm{m}) \geq \mathrm{d}\right\}\right]
\end{aligned}
$$

The reciprocal equatives in (44) below are analogous to the superlative sentences in (39). In the relevant reading, (44a) says that John ran exactly as fast as Mary and (44b), that it is exactly as cold in Berlin as it is in Frankfurt.

(44) a. John and Mary run equally fast.

b. It is equally cold in Berlin and Frankfurt.

In the analysis under consideration, these readings are due to the LFs in (45), which are isomorphic to those in (40), as the equative operator in (45) has moved in the same way as the superlative operator has in (40). Under Schwarz's (2007) analysis, then, reciprocal equatives are like superlatives under Heim's analysis in not being subject to an in situ requirement. Like superlatives, reciprocal equatives fail to be constrained by (what can be seen as) an analogue to Faraci's Generalization on too plus gapped infinitival.
a. [John and Mary] equally $\lambda \mathrm{d}[$ run $\mathrm{d}$ fast]
b. [Berlin and Frankfurt] equally $\lambda \mathrm{d} \lambda \mathrm{x}$ [it is $\mathrm{d}$ cold in $\mathrm{x}]$

In addition to allowing for interpretations parallel to interpretations excluded by Faraci's Generalization, superlatives and reciprocal equatives also fail to exhibit the frozen scope effect described for too plus gapped infinitival in section 3.1. Heim $(1999,2001)$ already showed that, much like too plus gapless infinitival, a superlative operator can be interpreted as taking inverse scope over certain intensional verbs. Sentence (46) illustrates this.

Mary wants to be richest.

The sentence has a surface scope reading that ascribes to Mary what could be characterized as a comparative desire, namely a desire to be richer than anyone else. But the sentence also has a reading consistent with Mary not having any such comparative desire. Specifically, it can be read as in (47a) below, asserting that there is some degree of wealth such that Mary is the only one who has a desire to reach it. In this reading, the sentence could be true by virtue of Mary wanting to be a millionaire, provided that everyone else is satisfied with a lesser degree of wealth. 

a. $\exists d\left[\forall \mathrm{w}^{\prime}\left[\mathrm{w}^{\prime} \in \mathrm{Bul}_{\mathrm{w}}(\mathrm{m}) \rightarrow \mathrm{RICH}_{\mathrm{w}}(\mathrm{m}) \geq \mathrm{d}\right] \& \sim \exists \mathrm{y}\left[\mathrm{y} \neq \mathrm{m} \& \forall \mathrm{w}^{\prime}\left[\mathrm{w}^{\prime} \in \mathrm{Bul}_{\mathrm{w}}(\mathrm{y})\right.\right.\right.$ $\left.\rightarrow \mathrm{RICH}_{\mathrm{w}},(\mathrm{y}) \geq \mathrm{d}\right]$ ]
b. Mary est $\lambda \mathrm{d}[$ wants [PRO to be $[\mathrm{d}$ rich] $]$

In Heim's analysis, the reading in question can be credited to a LF like (47b), where est takes inverse scope over the embedding verb want, targeting a position right below the matrix subject. So the superlative operator does not share with too plus gapped infinitival the requirement of being interpreted with surface scope.

The same is true for reciprocal equatives. Schwarz (2007) discusses examples like (48) below, which shows an ambiguity parallel to that of (46). The sentence has a surface scope interpretation, which ascribes somewhat unusual comparative desires to John and Mary, implying that each wants to be exactly as rich as the other. In addition, the sentence has a reading not reporting such comparative desires. It can be read as in (49a), which implies that the maximal degree to which John wants to be rich is also the maximal degree to which Mary wants to be rich. In this reading, the sentence could be true, for example, by virtue of each of John and Mary wanting to have a net worth of exactly one million dollars.

John and Mary want to be equally rich.
a. $\forall \mathrm{x}, \mathrm{y}\left[\mathrm{x}, \mathrm{y} \leq \mathrm{j}+\mathrm{m} \& \mathrm{x}, \mathrm{y} \in A\right.$ tom $\rightarrow\left\{\mathrm{d}:\left[\forall \mathrm{w}^{\prime}\left[\mathrm{w}^{\prime} \in \mathrm{Bul}_{\mathrm{w}}(\mathrm{x}) \rightarrow \mathrm{RICH}_{\mathrm{w}}(\mathrm{x}) \geq \mathrm{d}\right]\right\}=\right.$ $\left\{\mathrm{d}:\left[\forall \mathrm{w}^{\prime}\left[\mathrm{w}^{\prime} \in \mathrm{Bul}_{\mathrm{w}}(\mathrm{y}) \rightarrow \mathrm{RICH}_{\mathrm{w}}(\mathrm{y}) \geq \mathrm{d}\right]\right\}\right]$
b. [John and Mary] equally $\lambda \mathrm{d}[$ wants $[\mathrm{PRO}$ to be $[\mathrm{d}$ rich] $]$

This interpretation is derived by the LF in (49b), where the reciprocal equative operator takes inverse scope over want, landing right below the matrix subject. Like superlatives, then, reciprocal equatives participate in inverse scope readings unavailable to too plus gapped infinitival.

The observations presented above show that the frozen scope requirement and Faraci's in situ restriction on degree phrases with too' cannot be seen as more general constraints on two-place degree phrases. More accurately, the observations show this under the assumption that superlative and reciprocal equative degree phrases indeed are correctly analyzed as two-place. ${ }^{11}$ To be sure, it is not obvious that the two-place analysis given above are the only options. If these degree phrases could be reanalyzed as one-place, then the observations presented here would evidently be consistent with a general in situ constraint on two-place degree phrases. However, such a constraint would still lack independent empirical or conceptual motivation.

This calls into question the adequacy of the analysis of too plus gapped infinitival

\footnotetext{
${ }^{11}$ Under the so-called direct analysis of phrasal comparatives (e.g. Mary is taller than Bill) they also feature two-place degree phrases (Bhatt and Takahashi 2008, Heim 1985). Observations presented in Bhatt and Takahashi (2008) indicate that phrasal comparatives pattern with superlatives and reciprocal equatives in not being subject to constraints corresponding to the restrictions on too plus gapped infinitival.
} 
in terms of the type shifted operator too'. In section 4, we present an alternative account, one that makes non-subject gaps in infinitivals with too instances of the parasitic gap phenomenon.

\section{4. 'Parasitic' degree phrases and the in situ restriction}

In the last section, we pointed out two surprising restrictions on gapped degree phrases. These restrictions are unexpected under Chomsky's (1977) approach to the construction and suggest that our simple elaboration of that proposal is not correct. But if gapped degree phrases are not formed from a type-shifted variant of too that selects a gapped complement clause, what are they?

In this section we develop an alternative analysis which shares with the Chomskyan approach to too plus gapped infinitival the assumption that the antecedent for the gap in a degree phrase is a null operator. Where we depart from Chomsky is in our assumption about the landing site for operator movement. In particular, we propose that operator movement targets the edge of the degree phrase itself rather than too's infinitival complement. Thus we will replace a structure like (50) with the one in (51).

[DegP $\left[\right.$ too' $^{\prime}\left[\mathbf{O}_{\mathbf{i}}\left[\right.\right.$ for us to travel to $\left.\left.\left.\mathbf{t}_{\mathbf{i}}\right]\right]\right]$ [DegP $\mathbf{O}_{\mathbf{i}}$ [too [for us to travel to $\left.\left.\boldsymbol{t}_{\mathbf{i}}\right]\right]$ ]

In proposing to parse gapped degree phrases as null operator structures, that is, as structures of the form $O[\ldots]$, we propose to analyze them just like so-called parasitic gaps are analyzed in the separate antecedent approach (Browning 1987, Chomsky 1986, Contreras 1984, Nissenbaum 2000) outlined in section 1. We give canonical illustrations of parasitic gaps in (52), where the gap appears, respectively, in a clausal adjunct, in a subject DP, and in a non-subject DP. In the separate antecedent view of such parasitic gaps, the gapped adjunct clause and the gapped DPs in (52) are parsed as the null operator structures in (53).
a. this article, which ${ }_{\mathrm{i}}$ [we filed $\mathrm{t}_{\mathrm{i}}$ ] [without PRO reading _ ]
b. this theory, which $h_{i}$ [people who argue against $t_{j}$ ] [often end up adopting _ ]
c. this theory, which $I_{i}$ convinced [several proponents of _ ] to reject $t_{i}$
a. $\left[\mathrm{O}_{\mathrm{j}}\left[\right.\right.$ without $\mathrm{PRO}$ reading $\left.\left.\mathrm{t}_{\mathrm{j}}\right]\right]$
b. $\left[\mathrm{O}_{\mathrm{j}}\left[\right.\right.$ people who argue against $\left.\left.\mathrm{t}_{\mathrm{j}}\right]\right]$
c. $\left[\mathrm{O}_{\mathrm{j}}\left[\right.\right.$ several proponents of $\left.\left.\mathrm{t}_{\mathrm{j}}\right]\right]$

We will argue below that under the separate antecedent approach, central properties of gapped degree phrases can be made to fall out as special instances of the properties of parasitic gaps in general. In particular, building on Nissenbaum (2000), we will argue that the principles of semantic interpretation required to correctly compose null operator structures like those in (53) with their syntactic environment will also allow us to correctly compose structures containing gapped degree phrases like (51). Moreover, and crucially, we will argue that the restrictions identified in section 3 can be credited to constraints which, under the separate antecedent view, are needed independently to account for the 
behavior of canonical parasitic gaps of the sort shown in (52).

Below we will first address the question of how the null operator structures in (51) and (53) are semantically composed with their syntactic environment. Turning to the main point, we will then demonstrate that the curious restrictions on gapped degree phrases with too identified in the last section are instances of general constraints needed to regulate the distribution of parasitic gaps.

\subsection{Null operator structures and semantic composition}

In the Chomskyan approach to gapped degree phrases spelled out in section 2, we assumed that null operator movement triggers abstraction over an individual variable, creating a derived function from individuals. Nissenbaum (2000) makes the same assumption about null operator movement in his elaboration of the separate antecedent approach to parasitic gaps. We can rewrite the structures in (53) as shown in (54), continuing our practice of representing null operators as lambda binders in LFs. Correspondingly, we can rewrite the gapped degree phrase structure in (51) as shown in (55).
a. $\lambda x$ [without PRO reading $\mathrm{x}]$
b. $\lambda x[$ people who argue against $x]$
c. $\lambda x$ [several proponents of $\mathrm{x}]$

\section{$\lambda x[$ too [for us to travel to $\mathrm{x}]]$}

Each of the canonical parasitic gap examples in (52) features an instance of (relative clause creating) $w h$-movement in the main clause in addition to the null operator movement posited under the separate antecedent view. Nissenbaum (2000) adopts the standard view that, like null operator movement, overt wh-movement introduces lambda abstraction. Since the $w h$-movements in (52) target propositional nodes, they create derived predicates of individuals. Nissenbaum proposes that in parasitic gap constructions like those in (52), the null operator structure semantically composes with the predicate derived by $w h$-movement in the main clause.

The simplest kind of case is (52a), where the parasitic gap is inside an adjunct clause. Nissenbaum assumes that wh-movement in the main clause can target a position local to the attachment site of the adjunct clause. Taking this attachment site to be VP (for concreteness), this leads to a structure like (56), where the null operator structure syntactically combines with the predicate derived by wh-movement.

$$
\text { which }[\lambda x[\mathrm{vP} \text { John filed } \mathrm{x}] \lambda \mathrm{x}[\text { without PRO reading } \mathrm{x}]]
$$

In Nissenbaum's account, the two derived predicates in (56) compose via a process of generalized conjunction of the sort explicated in Partee and Rooth (1983). For the case at hand, conjunction of one-place predicate of individuals, generalized conjunction has much the effect of the familiar Predicate Modification rule formulated in Heim and Kratzer (1998). Assuming semantic vacuity of the relative pronoun, the semantic interpretation of (56) is derived by composing the semantic values in $(57 \mathrm{a}, \mathrm{b})$ into the conjoined property of individuals in (58). 
a. $\lambda \mathrm{x}_{\mathrm{e}} \cdot \lambda \mathrm{w}_{\mathrm{s}} \cdot \mathrm{j}$ filed $\mathrm{x}$ in $\mathrm{w}$

b. $\lambda x_{e} \cdot \lambda w_{s} \cdot j$ did not read $x$ in $w$

$\lambda x_{e} \cdot \lambda w_{s} \cdot j$ filed $x$ in $w \& j$ did not read $x$ in $w$

To be sure, gapped degree phrases (not to mention DPs containing parasitic gaps) cannot be interpreted in quite as simple a fashion. The derived function (55) certainly does not compose with its sister conjunctively. To accommodate such cases as well, we follow Nissenbaum (2000) in allowing a version of function application that is generalized in the same way that conjunction is generalized in Partee and Rooth (1983). To show how this can be done, we first spell out the rule of generalized conjunction hinted at above. In (59), we define a function CONJOIN that maps any two conjoinable semantic values to a third. 12

(59) a. Conjunction: If $A$ and $B$ are truth values, then $\operatorname{ConjoIN}(A, B)=1$ iff $A=B=1$.

b. Argument Identification: If $\mathrm{A}$ and $\mathrm{B}$ are conjoinable functions from the domain of type $\tau$, then $\operatorname{CONJOIN}(\mathrm{A}, \mathrm{B})=\lambda \mathrm{x}_{\tau} \cdot \operatorname{CONJOIN}(\mathrm{A}(\mathrm{x}), \mathrm{B}(\mathrm{x}))$.

The idea is, of course, that any two conjoinable denotations of sister constituents in a syntactic structure will be mapped to the denotation of their mother constituent by the function CONJOIN defined in (59). Clause (59a) ensures that generalized conjunction encompasses logical conjunction of truth values as a special case. By virtue of clause (59b), moreover, CONJOIN has in its domain not only pairs of truth values, but also pairs of functions to truth values, pairs of functions to functions to truth values, etc. In other words, CONJOIN has in its domain any two functions of the same type ending in t. In particular, as shown in (60) CONJOIN applies to the denotations of the two predicates in (56). Taking the denotations of those predicates to have type e(st), CONJOIN invokes Argument Identification twice: once for the individual argument position and once for the world argument position. Assuming that the predicates denote the particular e(st) functions in (57), the last line of (60) reduces to (58).

$\operatorname{CONJOIN}(\llbracket \lambda x[$ John filed $\mathrm{x}] \rrbracket, \llbracket \lambda x[$ without PRO reading $\mathrm{x}] \rrbracket)=$ by Argument Identification

$\lambda \mathrm{y}_{\mathrm{e}} \cdot \operatorname{CONJOIN}(\llbracket \lambda \mathrm{x}[$ John filed $\mathrm{x}] \rrbracket(\mathrm{y}), \llbracket \lambda \mathrm{x}[$ without PRO reading $\mathrm{x}] \rrbracket(\mathrm{y}))=$ by Argument Identification

$\lambda \mathrm{y}_{\mathrm{e}} \cdot \lambda \mathrm{w}_{\mathrm{s}} \cdot \operatorname{CONJOIN}(\llbracket \lambda \mathrm{x}[\operatorname{John}$ filed $\mathrm{x}] \rrbracket(\mathrm{y})(\mathrm{w}), \llbracket \lambda \mathrm{x}[$ without PRO reading $\mathrm{x}] \rrbracket(\mathrm{y})(\mathrm{w}))=$ by Conjunction

$\lambda \mathrm{y}_{\mathrm{e}} \cdot \lambda \mathrm{w}_{\mathrm{s} \cdot} \llbracket \lambda \mathrm{x}[\mathrm{John}$ filed $\mathrm{x}] \rrbracket(\mathrm{y})(\mathrm{w})=\llbracket \lambda \mathrm{x}[$ without PRO reading $\left.\mathrm{x}] \rrbracket(\mathrm{y})(\mathrm{w})\right)=1$

We suggest that in order to semantically compose structures containing gapped degree phrases like (55), the composition principle of function application must be

12 In the spirit of Partee and Rooth (1983), we take two semantic values A and B to be conjoinable (with each other) iff (i) both are truth values (type t), or (ii) both are functions from objects of any type $\tau$, such that for each $\mathrm{x}$ of type $\tau, \mathrm{A}(\mathrm{x})$ and $\mathrm{B}(\mathrm{x})$ are conjoinable (with each other). 
generalized in the same way as conjunction is generalized in (59), extending the role of Argument Identification in a way proposed in Nissenbaum (2000). We will show that this move will allow us to assign the intended interpretation to the LF in (61), where the gapped degree phrase in (55) occurs in situ.

\section{Berlin is $\lambda \mathbf{x}[$ too [for us to travel to $\mathbf{x}]]$ cold}

Under current assumptions, the gapped degree phrase has the denotation given in (62a), a function of type e $((\mathrm{d}(\mathrm{st})) \mathrm{st})$. Note that the outermost argument, of type e, is the argument introduced by null operator movement, while the remainder accords with the lexical entry for too in (16) above. As for the gradable adjective cold, we can assume that it has the denotation in (62b). Note that the function in (62b) has the semantic type $\mathrm{e}(\mathrm{d}(\mathrm{st})))$, which makes the individual argument outermost, an innocuous departure from our earlier view that gradable adjective denotations have type $\mathrm{d}((\mathrm{e}(\mathrm{st}))) .{ }^{13}$

$$
\begin{aligned}
& \text { a. } \lambda \mathrm{x}_{\mathrm{e}} \cdot \lambda \mathrm{f}_{\mathrm{d}(\mathrm{st})} \cdot \lambda \mathrm{w}_{\mathrm{s}} \cdot \exists \mathrm{d}\left[\mathrm{f}(\mathrm{d})(\mathrm{w}) \& \sim \exists \mathrm{w}^{\prime}\left[\mathrm{w}^{\prime} \in \mathrm{Acc}_{\mathrm{w}} \& \mathrm{f}(\mathrm{d})\left(\mathrm{w}^{\prime}\right) \& \text { we travel to } \mathrm{x} \text { in } \mathrm{w}^{\prime}\right]\right] \\
& \text { b. } \lambda \mathrm{x}_{\mathrm{e}} \cdot \lambda \mathrm{d}_{\mathrm{d}} \cdot \lambda \mathrm{w}_{\mathrm{s}} \cdot \operatorname{COLD}_{\mathrm{w}}(\mathrm{x}) \geq \mathrm{d}
\end{aligned}
$$

The two functions in (62) are not combinable by standard composition principles. Note, however, that the functions in the range of (62a), functions of type $(\mathrm{d}(\mathrm{st}))(\mathrm{st})$, have the functions in the range of (62b), functions of type $d(s t)$, in their domain. In other words, the two functions in (62) can be turned into straightforwardly composable semantic values by applying each to an individual argument. Specifically, the functions in (62) map their individual arguments to a generalized degree quantifier and a degree property, respectively, allowing for the latter to be fed as an input to the former.

This observation leads us to follow Nissenbaum (2000) in extending the definition in (59) in the manner shown in (63). There we define a function COMPOSE (replacing CONJOIN), which maps any two composable semantic values to a third. ${ }^{14}$ Assuming that (63) exhausts the ways denotations can be combine in semantic composition, we can use the COMPOSE function to state the general composition rule in (64).

(63) a. Conjunction: If $A$ and $B$ are truth values, then $\operatorname{compose}(A, B)=1$ iff $\mathrm{A}=\mathrm{B}=1$

b. Application: If $\mathrm{A}$ is a function whose domain contains $\mathrm{B}$, then $\operatorname{COMPOSE}(\mathrm{A}, \mathrm{B})$ $=\mathrm{A}(\mathrm{B}){ }^{15}$

c. Argument Identification: If $\mathrm{A}$ and $\mathrm{B}$ are composable functions from the domain of type $\tau$, then $\operatorname{COMPOSE}(\mathrm{A}, \mathrm{B})=\lambda \mathrm{x}_{\tau}$. $\operatorname{COMPOSE}(\mathrm{A}(\mathrm{x}), \mathrm{B}(\mathrm{x}))$.

13 With Rett (2008), we assume that our revised semantic type for gradable adjectives is consistent with the observed word order given that the subject moves to its surface position.

${ }^{14}$ We take two semantic values A and B to be composable (with each other) iff (i) both A and B are truth values (type $t$ ), or (ii) $A$ is a function and $B$ is in its domain, or (iii) both are functions from objects of any type $\tau$, such that for each $\mathrm{x}$ of type $\tau, \mathrm{A}(\mathrm{x})$ and $\mathrm{B}(\mathrm{x})$ are composable (with each other).

${ }^{15}$ Strictly speaking, we should say $\operatorname{COMPOSE}(\mathrm{A}, \mathrm{B})=\operatorname{COMPOSE}(\mathrm{B}, \mathrm{A})=\mathrm{A}(\mathrm{B})$, in order for our definition to interact appropriately with the composition rule (64), which does not make reference to linear order. 
(64) If $\alpha$ is a branching node with daughters $\beta$ and $\gamma$, then for any assignment $g, \llbracket \alpha \rrbracket^{g}=$ $\operatorname{COMPOSE}\left(\llbracket \beta \rrbracket^{\mathrm{g}}, \llbracket \gamma \rrbracket^{\mathrm{g}}\right)$.

Under these definitions, the LF in (61) is interpretable, and moreover yields intended denotation. In (65) we show how the COMPOSE function applies in successive steps to the denotations of the degree phrase and of the adjective in (61), invoking first a single step of Argument Identification, then a step of Application.

$$
\begin{aligned}
& \operatorname{COMPOSE}(\llbracket \lambda \mathbf{x}[\mathbf{t o o} \text { [for us to travel to } \mathbf{x}]] \rrbracket, \llbracket \operatorname{cold} \rrbracket)= \\
& \text { by Argument Identification } \\
& \lambda y \cdot \operatorname{COMPOSE}(\llbracket \lambda \mathbf{x}[\mathbf{t o o} \text { [for us to travel to } \mathbf{x}]] \rrbracket(y), \llbracket \operatorname{cold} \rrbracket(y))= \\
& \text { by Application } \\
& \lambda y \cdot \llbracket \lambda \mathbf{x}[\text { too }[\text { for us to travel to } \mathbf{x}] \rrbracket(\mathrm{y})(\llbracket \operatorname{cold} \rrbracket(\mathrm{y}))
\end{aligned}
$$

Assuming the denotations in (62), the last line of (65) reduces to (66). This function can then apply to Berlin to yield the proposition that it is colder in Berlin than it is in any accessible world in which we travel to Berlin.

$$
\lambda \mathrm{y}_{\mathrm{e}} \cdot \lambda \mathrm{w}_{\mathrm{s}} . \exists \mathrm{d}\left[\operatorname{COLD}_{\mathrm{w}}(\mathrm{y}) \geq \mathrm{d} \& \sim \exists \mathrm{w}^{\prime}\left[\mathrm{w}^{\prime} \in \mathrm{Acc}_{\mathrm{w}} \& \operatorname{COLD}_{\mathrm{w}^{\prime}}(\mathrm{y}) \geq \mathrm{d} \& \text { we travel to } \mathrm{y} \text { in } \mathrm{w}^{\prime}\right]\right]
$$

So far, what we have shown is that if a gapped degree phrase is a derived function formed by operator movement to its periphery, our generalized composition rule would allow it to compose with a degree adjective in a natural way and fill the gap appropriately with the adjective's subject.

Of course, as natural as the rule is, we would like to see that it has some additional application besides gapped degree phrases. In fact, Nissenbaum (2000) argued that under the separate antecedent approach to parasitic gaps, exactly this rule is needed to allow for semantic interpretation of parasitic gaps embedded in DPs, as in (52b-c). To see a case that is parallel to the gapped degree phrase, consider a parasitic gap that appears not in a VPadjunct but in a quantificational DP. A relevant example is (52c), whose structure is given in (67):

$$
\text { this theory, which I convinced }\left[\mathbf{O}_{j}\left[\text { several proponents of } t_{j}\right]\right] \text { to reject } t_{i}
$$

The sister of which in (68) is the relevant subpart of the LF of (67), formed by whmovement and quantifier raising of the parasitic DP:

$$
\text { which }[\lambda \mathbf{x} \text { [several proponents of } \mathbf{x}] \lambda x \lambda y[v p \text { we convinced } \mathbf{y} \text { to reject } \mathrm{x}]]
$$

Just as in the case considered above involving a gapped degree phrase, both the covertly moved null operator structure and its scope denote functions from individuals. Specifically, the null operator structure has a denotation of type e((e(st))st), that is, it denotes a function from individuals to generalized quantifiers. Its sister denotes a function of type e(e(st)), that is, a function from individuals to one-place properties, which are suitable arguments for generalized quantifiers. So, if each denotation were fed an individual, one of the resulting functions could take the other as an argument. The two denotations are therefore 
in the domain of COMPOSE. We make this transparent in the derivation in (69), which is parallel to (65) above.

$$
\begin{aligned}
& \operatorname{COMPOSE}(\llbracket \lambda \mathbf{x}[\text { several proponents of } \mathbf{x}]] \rrbracket, \llbracket \lambda x \lambda y[\text { we convinced } \mathbf{y} \text { to reject } \mathrm{x}] \rrbracket)= \\
& \text { by Argument Identification } \\
& \lambda z \text {.COMPOSE }(\llbracket \lambda \mathbf{x}[\text { several proponents of } \mathbf{x}]] \rrbracket(z), \llbracket \lambda x \lambda y[\text { we convinced } \mathbf{y} \ldots x] \rrbracket(z))= \\
& \text { by Application } \\
& \lambda z . \llbracket \lambda \mathbf{x}[\text { several proponents of } \mathbf{x}]] \rrbracket(z)(\llbracket \lambda x \lambda y[\text { we convinced } \mathbf{y} \text { to reject } x] \rrbracket \rrbracket(z))
\end{aligned}
$$

The last line of (69) denotes the intended one-place property of being an entity which we convinced several proponents of to reject-ensuring that the parasitic gap will be anaphoric to the gap left behind by the relative pronoun. ${ }^{16}$

\subsection{A syntactic restriction on parasitic constituents}

It seems, then, that if gapped degree phrases resemble canonical parasitic gap constructions in having null operator movement to the phrase-periphery, the resemblance is deeper than just this syntactic property. Both constructions would require the same generalized rule of semantic interpretation to compose with their sisters.

We propose that the similarity runs deeper still: 'Parasitic' degree phrases are subject to the very same set of syntactic constraints that limit the distribution of canonical parasitic gaps. This set of syntactic constraints is quirky and ill-understood, but it does provide a useful signature which can be used to diagnose seemingly disparate constructions as all belonging to the same family (such as parasitic gaps in VP adjunct and those embedded in subject or indirect object DPs). The aim of this section is to argue that the gapped degree phrase is indeed a member of that family.

\subsubsection{Three known constraints on Parasitic Gaps}

We noted in the introduction that the separate antecedent theory of parasitic gaps is in danger of overgenerating, and therefore needs to be supplemented with constraints on

\footnotetext{
${ }^{16}$ Notice that in (68), the lambda-operators in the null operator structure's scope appear in the reverse order of what would be expected under Heim and Kratzer's (1998) theory of chain formation (see footnote 10). In that theory, the lambda-binder introduced by movement is always prefixed to the scope of the moved constituent, while in (68) the lambda-binder introduced by the covert movement of the null operator structure appears below the lambda-binder introduced by movement of which. A structure identical to (68) but with the lambda-binders reversed would be interpretable by means of generalized function application (63), but the interpretation is not the one that is attested: it is impossible to interpret examples like (52b,c) with the gaps filled that way (i.e. with which as the indirect object of convinced in (52c)).

The Heim and Kratzer syntax of chain formation is straightforward, and evidence for it has been presented in a number of recent works (e.g. the references in footnote 10). As (68) indicates, the separate antecedent approach necessitates a principled exception to the Heim and Kratzer syntax of chain formation, the generalization being that a moved null operator structure places its lambda-binder just below the lambda introduced by the licensing wh-movement. The separate antecedent approach, then, remains incomplete until we come to understand the nature of this exception. Until this gap in the separate antecedent theory has been filled, there is room for doubt about the feasibility of the approach. However, we think that on balance, the evidence for the theory (including the arguments we adduce in this paper) is compelling.

Finally, it should be noted that this problem does not arise for gapped degree phrases like the one in (61).
} 
possible attachment sites of parasitic constituents - constraints that seem to be purely syntactic in nature. To illustrate, once we allow the needed generalized composition rules, examples like (70) cannot be blocked by semantic interpretability.
a. *John filed that article [without our talking to
b. *That politician convinced [several followers of
] to oppose the bill

The generalized composition rules should allow (70a) to have an interpretation in which the subject, John, antecedes the gap in the adjunct clause. The V' shown in (71a) is a (nonderived) predicate, and so would be able to compose with the parasitic adjunct by generalized conjunction if we assume an LF like (71b) to be available. No such interpretation is available, however, as the sentence is ungrammatical. Similarly, the subject of (70b) should be able to antecede the gap in the bracketed DP, if we assume that the latter can undergo quantifier raising from its surface position in (72a) to a position just below the subject of $V^{\prime}$, as shown in (72b). The raised parasitic DP could then compose with its sister in (72b) by generalized function application, if this LF were available. But here, too, the sentence is ungrammatical despite the semantic interpretability of the hypothesized LF structure. ${ }^{17}$

a. John [ $\mathrm{V}^{\prime}$ filed that article]

b. John $\left[v^{\prime}\right.$ filed that article] $\lambda \mathbf{x}$ [without our talking to $\left.\mathbf{x}\right]$
a. That politician $\left[\mathrm{v}^{\prime}\right.$ convinced $[\lambda \mathrm{x}[$ several followers of $\mathrm{x}]]$ to $\left.\ldots\right]$
b. That politician $\lambda \mathbf{x}$ [several followers of $\mathbf{x}] \lambda y\left[v^{\prime}\right.$ convinced $y$ to ...]

The ungrammaticality of examples like (70), then, suggests a constraint that prevents LFs such as (71b) and (72b) from being derived in the syntax. The relevant constraint would have to block both quantifier raising of the parasitic DP and attachment of the parasitic adjunct to $\mathrm{V}^{\prime}$. As far as we know, no satisfying explanation for this constraint has been offered, so we state it here as a yet-to-be-explained generalization:

The V' generalization: null operator structures cannot attach to V'.

Notice that there is in principle an alternate syntactic parse available for examples like (70), namely the structure in which the parasitic constituent is adjoined below the subject in its derived position. That potential attachment site, the $T^{\prime}$, would have the very same semantic type as the $\mathrm{V}^{\prime}$, and would consequently allow for an interpretation in which

\footnotetext{
${ }^{17}$ Notice that the interpretation that would result from (72b), perhaps somewhat counterintuitively, is one in which the convincing is done by the followers while the syntactic subject, the politician, is who they convince. This jarring reversal of semantic roles would stem from the fact that the lambda-binder introduced by quantifier raising of the parasitic DP $(\lambda y)$ has no choice but to go right after the raised phrasenotwithstanding the observation made in footnote 16. The reason is that in this example the target of movement (the $V^{\prime}$ ) is a non-derived predicate, and as such it has no syntactically visible lambda-binder that could end up preceding that of the parasitic DP, in a manner analogous to (68). Consequently, generalized function application would result in that politician binding the trace of the (parasitic) indirect object DP.
} 
the main-clause subject antecedes the parasitic gap. Specifically, movement of the subject to its surface position creates a derived function, illustrated in (74). That function could then compose with either a parasitic adjunct (by generalized conjunction) or with a raised parasitic DP (by generalized function application).

$$
\text { John } \lambda z\left[T^{\prime} \ldots \quad z\left[V^{\prime} \text { filed } \ldots\right]\right]
$$

Again, since the sentences are ungrammatical under either parse, in spite of their semantic interpretability, the very same examples motivate an additional constraint, one which would block this structure as well.

The $\mathbf{T}^{\prime}$ generalization: null operator structures cannot attach to $\mathrm{T}^{\prime}$.

In addition to the prohibition on forming LFs in which the subject antecedes the parasitic gap, there must be an additional constraint. Examples like (76) are ungrammatical, despite the fact that covert movement of the (italicized) quantificational DPs could create exactly the right kind of derived predicates that should allow parasitic gaps. As Engdahl (1983) observed, overt movement in the main clause seems to be required for parasitic gaps of the kind in (76).

(76) a. *John filed each of those articles [without our reading _ ]

b. *The politician convinced [several followers of _ _ to oppose each of those bills

In particular, quantifier raising of each of those articles in (76a) would derive the function shown in (77a), which should in principle then provide a suitable attachment site for the parasitic adjunct in (77b). Similarly, quantifier raising in (76b) would derive the function in (78a), which ought to provide a suitable landing site for quantifier raising of the parasitic DP as shown in (78b). Both LFs would be interpretable and would give rise to readings in which the raised universal DP antecedes the parasitic gap.

(77) a. [each of those articles] $\lambda z$ [John filed $z]$

b. [each of those articles] $\lambda z$ [John filed $z] \lambda \mathbf{x}$ [without our reading $\mathbf{x}$ ]

a. [each of those bills] $\lambda z[$ the pol. convinced $[\lambda x[$ several followers of $x]]$ to oppose z]

b. [each of those bills]

$\lambda \mathbf{x}$ [several followers of $\mathbf{x}] \lambda z \lambda y[$ the politician convinced $\mathbf{y}$ to oppose $z$ ]

The third constraint that is needed, then, would prohibit null operator structures from attaching between a covertly raised DP and its binder index.

(79) The LF-movement generalization: null operator structures cannot attach to predicates derived by covert movement.

Taken together, these three syntactic constraints are part of a signature pattern of parasitic gaps. Our analysis of gapped degree phrases as null operator structures, then, predicts that 
they will be subject to the same constraints. In the next subsection, we argue for precisely this result.

\subsubsection{The in situ restriction revisited}

Let us now return to the unexpected constraints on gapped degree phrases that we reported in section 3. We begin with Faraci's Generalization, according to which the gap in the degree phrase must be anaphoric to the subject of the adjective. This generalization excludes (80) because this adjective does not predicate any subject that could antecede the gap. The DP Mary, which might in principle be expected to antecede the gap, is the subject of the verb runs rather than of the adjective with which the degree phrase is in construction.

*Mary runs too fast for me to keep up with

Under current assumptions, the only way to have Mary antecede the gap would be for the degree phrase to move to a position just below the subject. There are two such positions: the degree phrase could target either the position just below the underlying position of the subject —i.e. the $\mathrm{V}^{\prime}(81 \mathrm{a})$ — or the position just below the subject's surface position — just below $\mathrm{T}^{\prime}(81 \mathrm{~b}) .{ }^{18}$
a. *Mary $\lambda \mathbf{x}$ [too [for me to keep up with $\mathbf{x}$ ]] $\lambda \mathrm{d}\left[\mathrm{v}^{\prime}\right.$ runs $\mathrm{d}$ fast]
b. *Mary $\lambda \mathbf{x}\left[\right.$ too [for me to keep up with $\mathbf{x}$ ]l $\lambda \mathrm{z} \lambda \mathrm{d}\left[\mathrm{T}^{\prime} \ldots \mathrm{z}\right.$ runs $\mathbf{d}$ fast]

But these two LFs violate, respectively, the $\mathrm{V}^{\prime}$ and $\mathrm{T}^{\prime}$ generalizations governing attachment sites for null operator structures. Given the independent support for these generalizations, therefore, the ungrammaticality of (80) follows from our assumption that gapped degree phrases are null operator structures.

Faraci's Generalization also excludes the interpretation of (82a) in which Mary antecedes the gap, as the DP is not the subject of the adjective but rather its internal argument. (82b) is excluded by Faraci's Generalization as well: Berlin cannot antecede the gap since it is not the subject of the adjective. These examples, too, would be expected to be grammatical on the relevant reading, if Mary and Berlin could raise covertly to derive a predicate for the gapped degree phrase to attach to.

(82) a. John is too angry at Mary for us to invite

b. *It is too cold in Berlin for us to travel to

The relevant structures are shown in (83), in which Mary and Berlin have covertly raised and in which the parasitic degree phrases target positions just below them. These LFs would be interpretable and would yield the relevant interpretations. However, they cannot

18 In the $\mathrm{V}^{\prime}$ case (81a), the lambda-binder introduced by raising of the degree phrase would again have no choice but to go over the $\mathrm{V}^{\prime}$ and hence outside the lambda-binder inherent to that (non-derived) predicate, as noted in footnote 17. Consequently, this LF would be ruled out for an additional reason, namely the resulting type mismatch (i.e. a function from degrees cannot compose with a function from individuals). The same consideration applies to example (86a) below. 
be derived in the syntax as they violate the LF movement generalization.
a. *Mary $\lambda \mathbf{x}$ [too [for us to invite $\mathbf{x}$ ]] $\lambda \mathrm{y} \lambda \mathrm{d}[\mathrm{John}$ is $\mathbf{d}$ [angry at $\mathrm{y}]$ ]
b. *Berlin $\lambda \mathbf{x}$ [too [for us to travel to $\mathbf{x}$ ]] $\lambda \mathrm{y} \lambda \mathrm{d}[$ it is [d cold] [in $\mathrm{y}]$ ]

In addition to Faraci's Generalization, we observed in Section 3.1 that gapped degree phrases cannot take inverse scope over an intensional operator (our Frozen Scope Generalization). In keeping with this generalization, (84) can only be interpreted on its surface scope reading (roughly paraphrased as $85 \mathrm{a}$ ), which entails that John wants not to be hired. The Frozen Scope Generalization excludes a reading that would allow John to have no objection to being hired $(85 \mathrm{~b})$, since such a reading would require the gapped degree phrase to have the matrix verb want in its scope.

John wants to be too rich [for the monastery to hire

a. In all of John's desire worlds, his degree of wealth is incompatible with the monastery's hiring him.

b. John's desired degree of wealth is incompatible with the monastery's hiring him

Just as with Faraci's Generalization above, the current approach should allow us in principle to construct suitable LFs that derive the unavailable wide scope reading. In particular, as long as the gapped degree phrase can target a position just below the matrix subject, the wide scope reading would arise. But, here just as above, the relevant LF would have to violate either the $\mathrm{V}^{\prime}$ or the $\mathrm{T}^{\prime}$ generalization:
a. *John $\lambda \mathbf{x}$ [too [for the monastery to hire $\mathbf{x}]] \lambda \mathrm{d}\left[\mathrm{V}^{\prime}\right.$ wants [PRO be $\mathrm{d}$ rich]]
b. *John $\lambda \mathbf{x}$ [too [for the monastery to hire $\mathbf{x}]] \lambda z \lambda d\left[\mathrm{~T}^{\prime} \ldots \mathrm{z}\right.$ wants [PRO be $\mathbf{d}$ rich]]

In Section 3 we contemplated an approach that relied on a type-shifted degree operator $t o o^{\prime}$. Under that approach, we took Faraci's Generalization and the Frozen Scope Generalization to point to an in situ constraint on the interpretation of gapped degree phrases. But the in situ constraint remained unexplained, there being no independent motivation for excluding the LF structures underlying the unattested readings. In fact, as we saw in section 3.4, other kinds of degree phrases do seem to be able to target the landing sites that are unavailable to gapped degree phrases with too, indicating that there is something quirky and exceptional about the distribution of the latter. On the current approach, the structures underlying the unavailable readings are isomorphic to the corresponding structures under the too' approach, modulo our new assumption about the internal structure of the degree phrases. The gapped degree phrases are now analyzed as null operator structures, and syntactic constraints on that type of structure that are independently motivated by the distribution of canonical parasitic gaps derive the effect of the previously unexplained restriction on gapped degree phrases. 
Note that our current approach allows us to derive the restriction without stipulating a general prohibition on raising gapped degree phrases. This leaves open the question whether gapped degree phrases can be raised to positions other than those where attachment of null operator structures are prohibited. In fact, there is an obvious kind of case to look at, namely the case in which the null operator structure attaches below an overtly moved $w h$-phrase - precisely the environment in which canonical parasitic gaps are licensed. In the next section we will show that in just such environments, gapped degree phrases are able to violate both Faraci's Generalization and the Frozen Scope Generalization.

\section{Exceptions}

We showed in the previous section that for all the examples considered so far, our hypothesis about the structure of gapped degree phrases can derive the effects of an in situ restriction on such degree phrases, which we had analyzed in section 3 as those headed by a type-shifted operator oo' $^{\prime}$. This is because the only potential landing sites in the examples we have considered are known to be independently prohibited for null operator structures. It is important to note, however, that null operator structures are not, in general, restricted to being interpreted in situ. This is shown most clearly by the very existence of gapped object DPs, which we do not assume to be interpreted in situ. Recall example (67), repeated here, whose LF involves quantifier raising of the gapped DP as shown in (68):

(67) this theory, which we convinced $\left[\mathbf{O}_{\mathbf{j}}\left[\right.\right.$ several proponents of $\left.\left.\mathbf{t}_{\mathbf{j}}\right]\right]$ to reject $t_{\mathrm{i}}$ (68) which $\lambda \mathbf{x}$ [several proponents of $\mathbf{x}] \lambda y \lambda z$ [we convinced $z$ to reject $y$ ]

In this case, raising of the null operator structure does not violate the generalizations noted in section 4.3. Rather than target $\mathrm{V}^{\prime}$ or $\mathrm{T}^{\prime}$, the gapped DP targets a predicate derived by overt $w h$-movement. Of course, canonical parasitic gaps are famously licensed by overt wh-movement, evidently even if the gapped phrase raises from its underlying position. Given our analysis, then, we expect the same to hold for gapped degree phrases - that is, we expect overt $w h$-movement to license raising of a gapped degree phrase from its surface position, yielding principled violations of both Faraci's Generalization and our Frozen Scope Generalization. We will now show that these expectations are borne out as well.

\subsection{Exceptions to Faraci's Generalization}

Repeated in (87) below are Faraci's (1974) examples in (29) above, which we presented as initial illustrations of Faraci's Generalization on too plus gapped infinitival. We noted that, since the adjectives fast and much do not predicate subjects that could conceivable antecede the gaps in the infinitivals, Faraci's Generalization correctly predicts the examples in (87) to be unacceptable. Under our current assumption, these examples are excluded by the prohibition on attaching a null operator structure to $\mathrm{V}^{\prime}$ or $\mathrm{T}^{\prime}$. 
a. *Mary runs too fast [for me to keep up with $\_$].
b. *Homer eats too much [for Jim to keep up with _ ]

However, as we predict, Faraci's Generalization is overly restrictive. This is illustrated by the examples in (88), which differ from Faraci's examples in (87) only in that the matrix subjects have been relativized.

a. Mary, who $\mathrm{t}_{\mathrm{i}}$ runs too fast [for me to keep up with _ ]

b. Homer, who $\mathrm{t}_{\mathrm{i}}$ eats too much [for Jim to keep up with

Being identical to their counterparts in (87) in terms of argument structure, the examples in (88) are incorrectly excluded by Faraci's Generalization. However, these examples are acceptable, with the gap in the infinitival understood anaphoric to the matrix subject. The gap in the infinitival is bound by the subject $w h$-operator, just like an overt pronoun can be bound by the $w h$-operator in the examples in (89).

a. Mary, who $t_{\mathrm{i}}$ runs too fast [for me to keep up with her]

b. Homer, who $t_{i}$ eats too much [for Jim to keep up with him]

These observations reveal that it is possible for a non-subject gap in an infinitival with too to take as its antecedent an expression that has undergone overt relative whmovement, irrespective of its place in argument structure. Apparently, it is only in the absence of overt wh-movement that Faraci's Generalization describes the facts correctly. This is precisely what our analysis predicts because $w h$-movement creates additional positions to which null operator structures are known to be able to attach. Thus we correctly expect the examples in (88) to have well-formed, interpretable LFs:

who $\lambda \mathbf{x}$ [too [for me to keep up with $\mathrm{x}] \mathrm{\lambda} \quad \lambda \mathrm{z} \lambda \mathrm{d}[\mathrm{z}$ runs $\mathbf{d}$ fast]

To further illustrate this pattern, we present some additional observations. First, whmovement has the same effect for the other two examples presented in section 3 as illustrations of Faraci's Generalization. Take again example (29), repeated here as (91a). Since Berlin is not the subject of cold, Faraci's Generalization correctly excludes it as a possible antecedent of the gap in the infinitival clause, leaving the gap without an antecedent.

a. *It is too cold in Berlin [for us to travel to _ ]

b. Berlin, which it is too cold in $t_{i}$ [for us to travel to

The acceptability of the relative clause in (91b), where the gap in the infinitival is interpreted as bound by the $w h$-phrase, illustrates again the ability of $w h$-movement to make available otherwise unavailable antecedents.

Recall also that sentence (31), repeated here as (92), does not permit Mary, the 
internal argument of angry, to antecede the gap in the infinitival. This is again in accordance with Faraci's Generalization and the syntactic restrictions which we have argued to derive it.

John is too angry at Mary [for us to invite _ _ ].

Sentence (93) shows, expectedly by now, that $w h$-movement can exceptionally make the internal argument of angry available as an antecedent of the gap in the infinitival. Again, the gap can be understood as being bound by the wh-phrase, hence as anaphoric to Mary.

Mary, $w_{h o} J_{i}$ ohn is too angry at $t_{i}$ [for us to invite

In further support of our characterization of the data, note that relative $w h$ movement is not alone in licensing parasitic gaps. Ordinary question-forming movment, topicalization, and Heavy NP Shift are among the types of movement known to license canonical parasitic gaps:

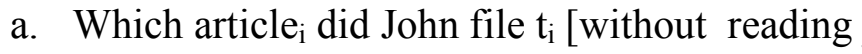
b. That article $e_{i}$, John filed $t_{i}$ [without reading
c. John filed $t_{i}$ [without reading __ ] [the dullest article we sent him $]_{i}$.

Correspondingly, we expect all of these kinds of A-bar movement as well to give rise to counterexamples to Faraci's Generalization. The examples in (95) illustrate that this expectation is borne out. These sentences are acceptable with the gap in the infinitival again understood as anaphoric to the DP that has been, respectively, questioned, topicalized and Heavey-NP-Shifted.

a. Which city $y_{i}$ is it too cold in $t_{i}$ [for us to travel to ]?

b. Mary $\mathrm{i}_{\mathrm{i}}$, John is too angry at $\mathrm{t}_{\mathrm{i}}$ [for us to invite

c. He showed us $t_{i}$ too quickly for us to write _ down [the name of his secret contact]

In this subsection we have identified a class of exceptions to Faraci's Generalization showing that, exactly as predicted, overt $w h$-movement can free gapped infinitivals with too from the requirement that the gap be anaphoric to the subject of the adjective combining with too.

\subsection{Exceptions to the Frozen Scope Generalization}

We observed in section 3 that a gapped degree phrase cannot take inverse scope over an intensional verb like want, to yield a reading that would otherwise be available were it not for the gap. For example, (96a) unambiguously implies that John does not want to be hired - an implication that we identified as a signature of the surface scope reading (i.e. 
with the degree phrase within the scope of want). In contrast, the gapless version (96b) can be understood as making no such implication, and can instead convey merely that the monastery's hiring policy is inconsistent with John's desired degree of wealth.
a. John wants to be too rich [for the monastery to hire
b. John wants to be too rich [for the monastery to hire him].

In the account given in section 4, the frozen scope generalization is not credited to a restriction on relative scope per se, but rather to a restriction on the possible landing sites of null operator structures such as gapped degree phrases. This again raises the question whether, in cases where a suitable landing site is made available by overt $w h$-movement, exceptions to the Frozen Scope Generalization will emerge. Once again, we expect exceptions to arise in just those environments where exceptions to Faraci's Generalization arise. Remarkably, this expectation is correct. Example in (97) is a case in point.

$$
\text { Mary, } w o_{i} \text { my mother wants me to be too angry at } t_{i} \text { [for me to (actually) dislike ] }
$$

This sentence is most naturally understood as conveying that what is incompatible with my disliking Mary is my mother's wanting me to be angry at Mary to the degree that she actually wants me to be angry at her. But note that this is not the one that would arise from the degree phrase taking surface scope below want. On its surface scope reading, this sentence conveys (implausibly) that in all of my mother's desire worlds, I am too angry at Mary to actually dislike her-that is, I am angry at Mary to degree that incompatible with me disliking her. The fact that the sentence has the more plausible reading paraphrased above indicates the availability of the LF (98), where the too' degree phrase scopes over want.

Mary, who $\lambda \mathbf{x}[$ too [for me to (actually) dislike $\mathbf{x}]] \quad \lambda \mathrm{y} \lambda \mathrm{d}[$ my mother wants me to be $\mathbf{d}$ [angry at $\mathrm{y}]$ ]

Consequently, we must conclude that the frozen scope observation described above is not in fact due to a restriction on the scope of too' plus gapped infinitival relative to other operators. Instead, it is due to a restriction on the possible landing site of the relevant degree phrases. Exactly as predicted, nothing in principle prevents gapped degree phrases from taking inverse scope over an intensional verb. Such degree phrases can covertly move past an intensional verb as long as they can target a position next to a predicate derived by overt wh-movement.

\section{Consequences for the theory of parasitic gaps}

In the preceding sections we have presented an analysis of gapped degree phrases as parasitic gaps, adopting the separate antecedent approach to the latter phenomenon. In doing so we have also, in effect, made an argument for that approach. In this section we 
will spell out the structure of that argument.

In section 3 we described two kinds of cases where gap in a degree phrase is impossible: cases in which the antecedent for the gap would be something other than the subject of the degree adjective, and cases in which the degree phrase takes inverse scope over an intensional operator. In section 5, we showed that overt wh-movement in the main clause leads to systematic exceptions to these restrictions. That is, overt $w h$-movement makes available antecedents other than the subject of the adjective, and allows the degree phrase to take inverse scope.

This pattern is transparently analogous to what is found with parasitic gaps, which are unavailable except when anaphoric to expressions which have undergone overt whmovement. It seems uncontroversial and inescapable, therefore, that the gaps in these cases - the ones presented in section 5-are actual parasitic gaps. However, taken in isolation, this conclusion would not tell us how to analyze parasitic gaps. In particular, it would not help us choose between the two types of theories sketched in the introduction. While we have analyzed the examples in section 5 in terms of the separate antecedent approach to parasitic gaps (i.e. with the gaps being traces of null operator movement, as in (99a)), we could just as easily have described these particular examples in terms of the shared antecedent approach as in (99b).

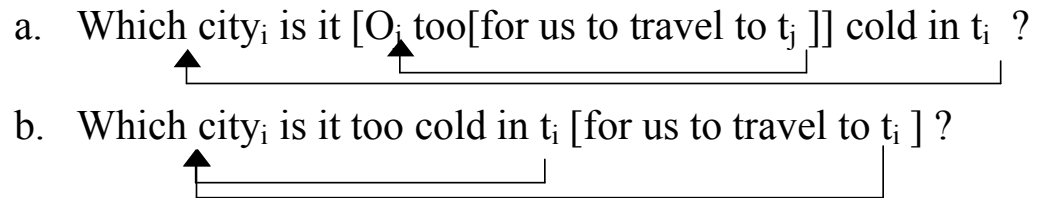

However, the two accounts come apart once we factor in the basic cases without wh-movement, such as the familiar example in (100):

$$
\text { John is too rich [for the monastery to hire _ _ ] }
$$

Under the separate antecedent approach, these examples, too, are analyzed as null operator structures. Consequently, they are expected to be restricted in the same way as parasitic gaps. In particular, gapped degree phrases are expected to be subject to whatever restrictions happen to govern possible attachment sites for null operator structures. We described three such restrictions in section 4, and showed that these were sufficient to explain the observable effects of Faraci's Generalization and the Frozen Scope Generalization.

Under the shared antecedent theory of parasitic gaps, in contrast, gapped degree phrases in the absence of $w h$-movement, as in (100), cannot be analyzed as parasitic. The shared antecedent theory, then, seems to require two separate analyses of gapped degree phrases, one for the exceptional cases in section 5, and one for more ordinary examples like (100). This theory remains silent about how such ordinary gapped degree phrases are to be analyzed. We presented one possibility in section 3 , namely our extension of Chomsky's (1977) theory involving the type-shifted operator $t o o^{\prime}$. But we showed there that this view would not explain the observable effects of Faraci's Generalization and the Frozen Scope Generalization. In fact, we are not aware of any way of deriving these restrictions other than the one we have proposed. Taking gapped degree phrases to be null 
operator structures appears to us to be the only way to derive the observed patterns.

In sum, we have presented an argument that parasitic gaps should be analyzed as null operator structures. In so doing we have also removed a central worry for that approach, namely that it falls short of predicting that parasitic gaps need movement in the matrix to be well-formed.

\section{Conclusions}

In this paper we have accomplished three main things. First, we reported a surprising empirical discovery, namely that (in the absence of matrix wh-movement) a gapped degree phrase is frozen in its surface scope position. Moreover, we showed that this Frozen Scope generalization would follow from the same in situ restriction that would give rise to Faraci's Generalization limiting the choice of antecedent for the gap in such degree phrases to the subject of the degree adjective. Second, we provided an explanation for this in situ restriction that also correctly predicts the exceptions that arise when there is $w h$-movement in the matrix clause. The explanation hinged on the observation that the exceptionally moved cases of gapped degree phrases have distributional characteristics identical to parasitic gaps, suggesting that they form a natural class. We argued that both gapped degree phrases and canonical parasitic gaps are null operator structures and consequently are governed not only by semantic interpretability but also by known syntactic restrictions on possible attachment sites. Lastly, since our explanation also rests on a uniform treatment of gapped degree phrases that remain in situ and those that raise in whmovement environments, we provided crucial support for a theory of parasitic gaps that does not require movement in the matrix clause - hence for the separate antecedent theory.

\section{References}

Barker, Chris: 2007, 'Parasitic Scope', Linguistics and Philosophy 30 (4), 407-444.

Beck, Sigrid: 2000. "Star Operators. Episode 1: Defense of the Double Star". In Kiyomi

Kusumoto and Elisabeth Villalta (eds): UMass Occasional Papers in Linguistics 23:

Issues in Semantics.Amherst, MA: GLSA, 1-23.

Bhatt, Rajesh, and Shoichi Takahashi: 2007, 'Direct comparisons: Resurrecting the direct analysis of phrasal comparatives' in Masayuki Gibson and Tova Friedman (eds.), Proceedings of SALT XVII.

Browning, Margaret: 1987, Null Operator Constructions, Doctoral Dissertation, MIT, MITWPL, Cambridge.

Chomsky, Noam: 1977, 'On Wh-Movment', in P. Culicover, T. Wasow and A. Akmajian (eds.), Formal Syntax, Academic Press, New York.

Chomsky, Noam: 1986, Barriers, MIT Press, Cambridge.

Contreras, Heles: 1984, 'A Note on Parasitic Gaps', Linguistic Inquiry 15, 698-701.

Cresswell, M. J.: 1976, 'The Semantics of Degree', in Barbara Partee (ed.), Montague

Grammar, Academic Press, New York, pp. 261-292.

Engdahl, Elisabet: 1983, 'Parasitic Gaps', Linguistics and Philosophy 6, 5-34.

Faraci, Robert: 1974, Aspects of the Grammar of Infinitives and for-Phrases, Doctoral Dissertation, MIT, MITWPL, Cambridge. 
Gawron, Jean Mark: 1995, 'Comparatives, Superlatives, and Resolution', Linguistics and Philosophy 18, 333-380.

Hacquard, Valentine: 2006, 'Aspects of Too and Enough constructions', in E. Georgala and J. Howell (eds.), Proceedings of SALT XV, Ithaca, NY: CLC Publications.

Heim, Irene: 1985, 'Notes on comparatives and related matters', ms., University of Texas, Austin.

Heim, Irene: 1999, 'Notes on Superlatives', ms., MIT.

Heim, Irene: 2001, 'Degree Operators and Scope', in C. Féry and W. Sternefeld (eds.), Audiatur Vox Sapientiae, Akademie-Verlag, Berlin.

Heim, Irene, and Angelika Kratzer: 1998: Interpretation in Generative Grammar, Blackwell, Cambridge.

Hornstein, Norbert, and Jairo Nunes: 2002, 'On asymmetries between parasitic gap and across the board movement constructions', Syntax 5 (1): 26-54.

Kayne, Richard: 1983, 'Connectedness', Linguistic Inquiry 14: 223-249.

Meier, Cécile: 2003, 'The Meaning of too, enough, and so...that', Natural Language Semantics 11 (1), 69-107.

Nissenbaum, Jon: 1998, 'Movement and derived predication: evidence from parasitic gaps', in U. Sauerland and O. Percus (eds.), The Interpretive Tract, MIT Working Papers in Linguistics, Cambridge, MA.

Nissenbaum, Jon: 2000, Investigations of Covert Phrase Movement, Doctoral Dissertation, MIT, MITWPL, Cambridge.

Nissenbaum, Jon and Bernhard Schwarz: 2008, "Two Puzzles about Infinitivals with Too", in Natasha Abner, Jason Bishop, and Kevin Ryan (eds.), Proceedings of the 27th West Coast Conference on Formal Linguistics, Somerville, MA: Cascadilla Proceedings Project, 344-352.

Nunes, Jairo: 1995, The copy theory of movement and linearization of chains in the minimalist program, Doctoral Dissertation, University of Maryland, College Park.

Partee, Barbara H., and Rooth, Mats: 1983, Generalized conjunction and type ambiguity. In Meaning, Use, and Interpretation of Language, eds. Rainer Bäuerle, Christoph Schwarze and Arnim von Stechow, 361-383. Berlin: de Gruyter.

Pesetsky, David: 1982, Paths and Categories. Doctoral Dissertation, MIT, MITWPL, Cambridge.

Rett, Jessica: 2008, 'Antonymy and Evaluativity', in M. Gibson and T. Friedman (eds.), Proceedings of SALT XVII, CLC Publications.

Sauerland, Uli: 1998, 'Plurals, derived predicates, and reciprocals', in U. Sauerland and O. Percus (eds.), The interpretive tract, MIT Working Papers in Linguistics, Cambridge, MA: 177-204.

Schwarz, Bernhard: 2007, 'Reciprocal Equatives', in E. Puig-Waldmüller (ed.), Proceedings of Sinn und Bedeutung 11, Universitat Pompeu Fabra, Barcelona, 568-582.

Schwarzchild, Roger: 2008, 'The semantics of comparatives and other degree constructions' Language and Linguistics Compass 2 (2), 308-331. 
Stechow, Arnim von: 1984, 'Comparing Semantic Theories of Comparison', Journal of Semantics 3, 1-177.

Stechow, Arnim von, Sveta Krasikova, and Doris Penka: 2004, "The meaning of German um zu: necessary condition and enough/too", handout for Workshop on Modal Verbs and Modality, University of Tübingen. 